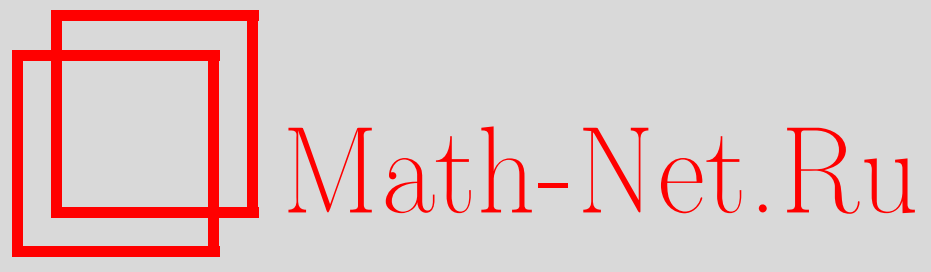

В. С. Жгун, Д. В. Миронов, Разделяющие системы камер Вейля, Матем. заметки, 2007, том 82, выпуск $2,310-314$

DOI: https://doi.org/10.4213/mzm3800

Использование Общероссийского математического портала Math-Net.Ru подразумевает, что вы прочитали и согласны с пользовательским соглашением http://www . mathnet.ru/rus/agreement

Параметры загрузки:

IP : 35.174 .16 .151

26 апреля 2023 г., 14:06:31

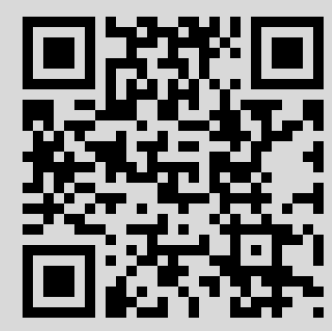




\section{Разделяющие системы камер Вейля}

\section{В. Жгун, Д. Миронов}

В связи с исследованием некоторых специальных действий в [1] были введены понятия индекса разделения (separation index) $\operatorname{sep}(\Delta)$ неприводимой системы корней $\Delta$ и, более общо, индекса разделения $\operatorname{sep}(K)$ неприводимой конечной линейной группы $K$, порожденной отражениями конечномерного вещественного пространства $L$. В [1] было доказано, что $\operatorname{rk}(\Delta)+1 \leqslant \operatorname{sep}(\Delta) \leqslant\left|W_{\Delta}\right|$, где $W_{\Delta}-$ группа Вейля системы корней $\Delta$ и, соответственно, $\operatorname{dim}(L)+1 \leqslant \operatorname{sep}(K) \leqslant|K|$, а также вычислены $\operatorname{sep}(\Delta)$ и $\operatorname{sep}(K)$ в размерностях, не превосходящих 2 . В настоящей работе получены более точные верхние оценки числа $\operatorname{sep}(\Delta)$.

Напомним основное определение. Далее мы считаем $\Delta$ подмножеством рационального векторного пространства $V$, являющегося линейной оболочкой $V$.

ОПРедЕЛЕНИЕ 1 . Подмножество $S$ множества камер Вейля системы корней $\Delta$ называется разделяющей системой камер Вейля, если выполняется следующее условие: для всякой ненулевой линейной функции $l \in V^{*}$ существует камера Вейля $C$ из $S$ такая, что

$$
\left.l\right|_{\bar{C}} \geqslant 0, \quad \bar{C} \cap \operatorname{Ker} l=\{0\}
$$

(через $\bar{C}$ обозначено замыкание $C$ ).

В [1] доказано, что разделяющие системы камер Вейля существуют (в этом утверждении существенно, что система $\Delta$ неприводима).

ОПРедЕЛЕниЕ 2 [1]. Индексом разделения $\operatorname{sep}(\Delta)$ системы корней $\Delta$ называется минимум чисел элементов в разделяющих системах камер Вейля системы корней $\Delta$.

Введем необходимые обозначения:

1) $C$ - фиксированная доминантная камера Вейля;

2) $\Delta^{+}$- система положительных корней в $\Delta$, определенная $C$;

3) $\Pi=\left\{\alpha_{1}, \ldots, \alpha_{n}\right\}-$ множество простых корней в $\Delta^{+}$;

4) $\pi_{i}$ - фундаментальный вес, двойственный $\alpha_{i}$;

5) мы считаем, что в $V$ зафиксирована структура евклидова пространства, определенная $W$-инвариантным скалярным произведением $\langle\cdot, \cdot\rangle$;

6) $H_{\alpha}$ - гиперплоскость в $V$, ортогональная $\alpha \in \Delta$;

7) $s_{\alpha}$ - отражение с зеркалом $H_{\alpha}$, переводящее $\alpha$ в $-\alpha$.

С этого момента для упрощения изложения мы называем камерой Вейля замыкание (по определению, открытой) камеры Вейля, из которого удалена точка $\{0\}$.

Сначала дадим описание индуктивного процесса построения разделяющих систем камер Вейля. Индукция ведется по рангу системы корней $\Delta$. База индукции очевидна: в случае $\mathrm{A}_{1}$ имеется только две камеры Вейля и набор будет состоять из них обеих.

Выберем некоторый простой корень $\alpha$. Пусть $\Delta_{\alpha}=\Delta \cap H_{\alpha}$. Выберем множество $\Delta_{\alpha}^{+}$ положительных корней подсистемы $\Delta_{\alpha}$ так, что $\Delta_{\alpha}^{+} \subset \Delta^{+}$. Группу Вейля системы корней $\Delta_{\alpha}$ мы обозначим $W_{\alpha}$ и будем рассматривать ее как подгруппу группы Вейля $W$, порожденную отражениями $s_{\beta}$, где $\beta \in \Delta_{\alpha}$ (заметим, что эта группа стабилизирует $\alpha$ ).

Теперь рассмотрим следующие два случая.

Работа выполнена при поддержке гранта 05-01-02805-CNRSL_а.

(C) В. Жгун, Д. Миронов, 2007 
(i) Система корней $\Delta_{\alpha}$ неприводима, и ее ранг на 1 меньше, чем у $\Delta$. Обозначим через $C_{\alpha}$ доминантную камеру Вейля системы $\Delta_{\alpha}$. Заметим, что $C_{\alpha} \supseteq C \cap H_{\alpha}$. Рассмотрим разделяющую систему камер Вейля для системы $\Delta_{\alpha}$. Камеры из этой системы получаются из $C_{\alpha}$ применением некоторых элементов из $W_{\alpha}$. Обозначим множество всех этих элементов через $W_{\alpha}^{\text {sep }}$.

(ii) Условие (i) не выполнено. Положим

$W_{\alpha}^{\text {sep }}=\left\{w \in W \mid \operatorname{dim} w C \cap H_{\alpha}=\operatorname{dim} H_{\alpha}, w C\right.$ и $C$ лежат по одну сторону от $\left.H_{\alpha}\right\}$.

Легко видеть, что в случае (ii)

$$
H_{\alpha}=\bigcup_{w \in W_{\alpha}^{\text {sep }}}\left(w C \cap H_{\alpha}\right) .
$$

(Это следует из того, что ни одна из камер Вейля не может пересекаться по своей внутренности с $H_{\alpha}, W$ действует на камерах транзитивно и, если две камеры имеют общую грань коразмерности 1 на гиперплоскости $H_{\alpha}$, то они переводятся друг в друга отражением $s_{\alpha}$ ).

В этом случае нам понадобится

Лемма 3. Рассмотрим случай (ii). Пусть $l$ - ненулевая линейная функция на $H_{\alpha}$. Тогда найдется элемент $w \in W_{\alpha}^{\text {sep }}$ такой, что $l$ строго положительна на $w C \cap H_{\alpha}$.

ДоказАтельство. Существует такой вектор $\chi \in H_{\alpha}$, что $l=\langle\chi, \cdot\rangle$. Ввиду (1) существует такой элемент $w \in W_{\alpha}^{\text {sep }}$, что $\chi \in w C \cap H_{\alpha}$. Тогда согласно следующей лемме $l(\lambda)=\langle\chi, \lambda\rangle>0$ для любого $\lambda \in w C \cap H_{\alpha}$.

ЛЕмма 4. Если $\chi_{1}, \chi_{2}$ - (ненулевые) векторы из $V$, лежащие в одной камере Вейля, $m o\left\langle\chi_{1}, \chi_{2}\right\rangle>0$.

Доказательство. Ввиду $W$-инвариантности $\langle\cdot, \cdot\rangle$ можно считать, что $\chi_{1}, \chi_{2} \in C$, так что $\chi_{j}=\sum_{\beta \in \Pi} c_{j \beta} \pi_{\beta}$, где $c_{j \beta} \geqslant 0$. Так как $\Delta$ неприводима, то (см., например, [2; таблица 2 , с. 308-309]) $\left\langle\pi_{\beta}, \pi_{\gamma}\right\rangle>0$ для всех $\beta, \gamma \in \Pi$. Это дает

$$
\left\langle\chi_{1}, \chi_{2}\right\rangle=\sum_{\beta, \gamma \in \Pi} c_{1 \beta} c_{2 \gamma}\left\langle\pi_{\beta}, \pi_{\gamma}\right\rangle>0 .
$$

Докажем теперь основное утверждение работы.

Теорема 5. Пусть $C_{0}$ - камера Вейля, содержащая корень $\alpha$. Тогда $C_{0},-C_{0}, w C$, $s_{\alpha} w C$, где $w$ пробегает множество $W_{\alpha}^{\mathrm{sep}},-$ разделяющая система камер Вейля для системы корней $\Delta$.

ДоказАтельство. Рассмотрим ненулевую линейную функцию $l_{\chi}(\cdot)=\langle\chi, \cdot\rangle$, где $\chi \in V$. Имеем $\chi=c \alpha+\tilde{\chi}$, где $c \in \mathbb{Q}, \tilde{\chi} \in H_{\alpha}$. Тогда $l_{\tilde{\chi}}(\cdot)=\langle\tilde{\chi}, \cdot\rangle-$ линейная функция, на плоскости $H_{\alpha}$. Рассмотрим сначала случай $\tilde{\chi} \neq 0$.

Рассмотрим ситуацию (i). Из того, что мы выбрали разделяющий набор камер для системы $\Delta_{\alpha}$ следует, что найдется элемент $w \in W_{\alpha}^{\text {sep }}$ такой, что функция $l_{\tilde{\chi}}(\cdot)$ строго положительна на камере $w C_{\alpha}$.

Пусть $c \geqslant 0$. Докажем, что тогда $l_{\chi}(\cdot)$ положительна на $w C$. Действительно, $w \bar{C}=$ $w \bar{C}_{\alpha}+\mathbb{Q}_{+} w \pi_{\alpha}$, где $\mathbb{Q}_{+}=\{a \in \mathbb{Q} \mid a \geqslant 0\}$. Для любого $v \in w \bar{C}$ имеем разложение $v=v_{\alpha}+a w \pi_{\alpha}$, где $v_{\alpha} \in w C \cap H_{\alpha} \subseteq w \bar{C}_{\alpha}$, а $a \geqslant 0$. Заметим, что $w$ стабилизирует $\alpha$, откуда

$$
\left\langle\alpha, w \pi_{\alpha}\right\rangle=\left\langle w^{-1} \alpha, \pi_{\alpha}\right\rangle=\left\langle\alpha, \pi_{\alpha}\right\rangle>0 .
$$

Тогда имеем следующую цепочку равенств:

$$
\begin{aligned}
l_{\chi}(v)=\left\langle c \alpha+\tilde{\chi}, v_{\alpha}+a w \pi_{\alpha}\right\rangle & =\left\langle\tilde{\chi}, v_{\alpha}\right\rangle+a c\left\langle\alpha, w \pi_{\alpha}\right\rangle+a\left\langle\tilde{\chi}, w \pi_{\alpha}\right\rangle \\
& =\left\langle\tilde{\chi}, v_{\alpha}\right\rangle+a\left\langle\tilde{\chi}, w \pi_{\alpha}\right\rangle+a c \frac{\langle\alpha, \alpha\rangle}{2}>0,
\end{aligned}
$$

так как $\left\langle\alpha, v_{\alpha}\right\rangle=0,\left\langle\tilde{\chi}, v_{\alpha}\right\rangle>0$, а также $\left\langle\tilde{\chi}, w \pi_{\alpha}\right\rangle \geqslant 0$. 
Докажем последнее неравенство. В случае (i) мы выбрали $w$ таким образом, что функция $\langle\widetilde{\chi}, \cdot\rangle$ строго положительна на камере $w C_{\alpha}$. Отсюда следует, что $\widetilde{\chi}$ принадлежит конусу $\sum_{\beta \in w \Delta_{\alpha}^{+}} \mathbb{Q}_{+} \beta$, натянутому на положительные корни из $w \Delta_{\alpha}^{+}$. В частности, $\tilde{\chi} \in \sum_{\beta \in w \Delta^{+}} \mathbb{Q}_{+} \beta$, а значит, функция $\langle\tilde{\chi}, \cdot\rangle$ положительна на камере Вейля $w C$. Учитывая, что $w \pi_{\alpha}-$ ребро камеры Вейля $w C$, получаем требуемое.

Пусть $c<0$. Докажем, что тогда $l_{\chi}(\cdot)$ положительна на камере $s_{\alpha} w C$. Аналогично предыдущему случаю имеем разложение $v=v_{\alpha}+a s_{\alpha} w \pi_{\alpha}$, где $v \in w C, v_{\alpha} \in s_{\alpha} w C_{\alpha}$, $a \geqslant 0$. Также имеет место цепочка равенств

$$
\left\langle\alpha, s_{\alpha} w \pi_{\alpha}\right\rangle=\left\langle w^{-1} s_{\alpha} \alpha, \pi_{\alpha}\right\rangle=-\left\langle\alpha, \pi_{\alpha}\right\rangle<0 .
$$

В этом случае аналогично предыдущему случаю имеем

$$
\begin{aligned}
l_{\chi}(v)=\left\langle c \alpha+\tilde{\chi}, v_{\alpha}+a s_{\alpha} w \pi_{\alpha}\right\rangle & =\left\langle\tilde{\chi}, v_{\alpha}\right\rangle+a c\left\langle\alpha, s_{\alpha} w \pi_{\alpha}\right\rangle+a\left\langle\tilde{\chi}, s_{\alpha} w \pi_{\alpha}\right\rangle \\
& =\left\langle\tilde{\chi}, v_{\alpha}\right\rangle+a\left\langle\tilde{\chi}, s_{\alpha} w \pi_{\alpha}\right\rangle-a c \frac{\langle\alpha, \alpha\rangle}{2}>0,
\end{aligned}
$$

так как $\left\langle\widetilde{\chi}, v_{\alpha}\right\rangle>0$ и $\left\langle\widetilde{\chi}, s_{\alpha} w \pi_{\alpha}\right\rangle \geqslant 0$ (последнее неравенство совершенно аналогично неравенству из предыдущего случая).

В случае (ii) ввиду (1) элемент $\widetilde{\chi}$ лежит в некоторой камере Вейля $w C$ системы $\Delta$. Без ограничения общности можно считать, что $\tilde{\chi} \in C_{\alpha}=C \cap H_{\alpha}$. Как и в случае (i), имеем разложение $v=v_{\alpha}+a \pi_{\alpha}$, где $v \in C, v_{\alpha} \in C_{\alpha}$, а $a \geqslant 0$. По лемме 3 выполнено неравенство $\left\langle\widetilde{\chi}, v_{\alpha}\right\rangle>0$.

Отсюда мы получаем при $c \geqslant 0$ :

$$
\begin{aligned}
l_{\chi}(v)=\left\langle c \alpha+\tilde{\chi}, v_{\alpha}+a \pi_{\alpha}\right\rangle & =\left\langle\tilde{\chi}, v_{\alpha}\right\rangle+a c\left\langle\alpha, \pi_{\alpha}\right\rangle+a\left\langle\tilde{\chi}, \pi_{\alpha}\right\rangle \\
& =\left\langle\tilde{\chi}, v_{\alpha}\right\rangle+a\left\langle\tilde{\chi}, \pi_{\alpha}\right\rangle+a c \frac{\langle\alpha, \alpha\rangle}{2}>0 .
\end{aligned}
$$

Нами было использовано неравенство $\left\langle\widetilde{\chi}, \pi_{\alpha}\right\rangle \geqslant 0$, которое следует из леммы 4 , примененной к $\pi_{\alpha}$ и весу $\tilde{\chi} \in C$.

При $c<0$ рассмотрим камеру $s_{\alpha} C$. Имеем разложение $v=v_{\alpha}+a s_{\alpha} \pi_{\alpha}$, где $v \in C$, $v_{\alpha} \in C_{\alpha}$, а $a \geqslant 0$. И нужное утверждение следует из неравенства

$$
l_{\chi}(v)=\left\langle\tilde{\chi}, v_{\alpha}\right\rangle+a c\left\langle\alpha, s_{\alpha} \pi_{\alpha}\right\rangle+a\left\langle\tilde{\chi}, s_{\alpha} \pi_{\alpha}\right\rangle=\left\langle\tilde{\chi}, v_{\alpha}\right\rangle-a c \frac{\langle\alpha, \alpha\rangle}{2}+a\left\langle\widetilde{\chi}, s_{\alpha} \pi_{\alpha}\right\rangle>0 .
$$

Осталось разобрать случай, когда $\tilde{\chi}=0$. Докажем, что функция $l_{\chi}(\cdot)$ строго положительна на камере $C_{0}$, либо на камере $-C_{0}$. Действительно, в этом случае $l_{\chi}(\cdot)=c\langle\alpha, \cdot\rangle$. Замечая, что $\alpha \in C_{0}$, мы снова можем воспользоваться леммой 4.

Таким образом, мы получили следующую оценку для индекса разделения.

СледСтвиЕ 6. Выполнена оценка $\operatorname{sep}(\Delta) \leqslant 2\left|W_{\alpha}^{\mathrm{sep}}\right|+2$.

С помощью этого следствия мы получаем ниже оценки на $\operatorname{sep}(\Delta)$ для каждого из типов систем простых корней

$$
\mathrm{A}_{l}, \quad \mathrm{~B}_{l}, \quad \mathrm{C}_{l}, \quad \mathrm{D}_{l}, \quad \mathrm{~F}_{4}, \quad \mathrm{E}_{6}, \quad \mathrm{E}_{7}, \quad \mathrm{E}_{8}
$$

(для $\Delta=\mathrm{G}_{2}$ и группы диэдра индекс разделения вычислен в [1]). Нам понадобится

Лемма 7. Пусть $\Delta$ - простая система корней одного из типов

$$
\mathrm{A}_{n}, \quad \mathrm{D}_{n}, \quad \mathrm{E}_{6}, \quad \mathrm{E}_{7}, \mathrm{E}_{8} .
$$

Тогда число камер Вейля, пересекающих $H_{\alpha}$ по грани коразмерности 1, равно $\operatorname{rk}(\Delta) \cdot\left|W_{\alpha}\right|$. 
ДоказАтельство. Пусть $C_{\beta}$ - грань коразмерности 1 камеры $C$, перпендикулярная простому корню $\beta \in \Pi$ соответственно. Как известно, группа Вейля действует транзитивно на системах корней указанных типов (доказательство сразу сводится к случаю $\mathrm{A}_{2}$, в котором оно очевидно). Поэтому найдется такой элемент $w \in W$, что $w C_{\beta} \in H_{\alpha}$. Будем называть $w C_{\beta}$ гранью типа $\beta$.

Заметим, что если $w_{1}, w_{2} \in W$ - такие элементы, что $w_{i} C_{\beta} \subset H_{\alpha}, i=1,2$, то $w_{1} w_{2}^{-1} \in$ $W_{\alpha}$. (Действительно, $w_{1} w_{2}^{-1}$ переводит грань $w_{2} C_{\beta}$ в грань $w_{1} C_{\beta}$, а так как они обе телесны в гиперплоскости $H_{\alpha}$, то этот элемент переводит в себя и $H_{\alpha}$.)

Из вышесказанного получаем, что камеры Вейля могут пересекать плоскость по грани коразмерности 1 любого типа $\beta \in \Pi$ и любые две камеры, пересекающие $H_{\alpha}$ по граням одного типа, могут быть переведены друг в друга элементом $W_{\alpha}$. Тем самым, их количество равно $\mathrm{rk}(\Delta)\left|W_{\alpha}\right|$.

(1) Пусть $\Delta$ - система корней типа $\mathrm{A}_{l}$. Тогда $|W|=(l+1)$ !. Выберем $\alpha=\varepsilon_{1}-\varepsilon_{2}$. Имеет место случай (ii), так как $\Delta_{\alpha}=\mathrm{A}_{l-2} . W_{\alpha} \cong S_{l-1}$ и, значит,

$$
\operatorname{sep}\left(\mathrm{A}_{l}\right) \leqslant 2 \operatorname{rk}(\Delta)\left|W_{\alpha}\right|+2=2 l !+2 .
$$

(2) Пусть $\Delta$ - система корней типа $\mathrm{B}_{l}$. Выберем $\alpha=\varepsilon_{l}$. В этом случае $\Delta_{\alpha}=\mathrm{B}_{l-1}$ (это те корни, в запись которых не входит $\varepsilon_{l}$ ). Таким образом, имеет место случай (i) и выполнена индуктивная оценка

$$
\left|W_{\mathrm{B}_{l}}^{\mathrm{sep}}\right|=2\left|W_{\mathrm{B}_{l-1}}^{\mathrm{sep}}\right|+2 .
$$

Откуда по индукции легко получить оценку

$$
\operatorname{sep}\left(B_{l}\right) \leqslant 2^{l+1}-2 .
$$

(3) Пусть $\Delta$ - система корней типа $\mathrm{C}_{l}$. Она двойственна системе корней $\mathrm{B}_{l}$, так что $W\left(\mathrm{C}_{l}\right)=W\left(\mathrm{~B}_{l}\right)$. Поэтому

$$
\operatorname{sep}\left(C_{l}\right)=\operatorname{sep}\left(B_{l}\right) \leqslant 2^{l+1}-2 .
$$

(4) Пусть $\Delta$ - система корней типа $\mathrm{D}_{l}$. В этом случае возьмем $\alpha=\varepsilon_{l-1}-\varepsilon_{l}$. Тогда $\Delta_{\alpha}=\mathrm{D}_{l-2} \times \mathrm{A}_{1}$, где система $\mathrm{A}_{1}$ порождена корнем $\varepsilon_{l-1}+\varepsilon_{l}$. Мы находимся в ситуации пункта (ii) (система $\Delta_{\alpha}$ приводима), поэтому

$$
\operatorname{sep}\left(\mathrm{D}_{l}\right) \leqslant 2|\Pi| \cdot\left|W_{\mathrm{D}_{l-2}}\right| \cdot\left|W_{\mathrm{A}_{1}}\right|+2=\frac{2^{l-1} l !}{(l-1)}+2 .
$$

(5) Пусть $\Delta$ - система корней типа $\mathrm{F}_{4}$. Если выбрать $\alpha=\varepsilon_{4}$, то $\Delta_{\alpha}=\mathrm{B}_{3}$. Мы находимся в ситуации случая (i). Воспользовавшись следствием 6 и случаем (2), получаем

$$
\operatorname{sep}\left(\mathrm{F}_{4}\right) \leqslant 30
$$

(6) Пусть $\Delta$ - система корней типа $\mathrm{E}_{6}$. Выберем $\alpha=\varepsilon_{1}-\varepsilon_{2}$. Тогда $\Delta_{\alpha}=\mathrm{A}_{5}\left(\varepsilon_{4}+\varepsilon_{5}+\right.$ $\varepsilon_{6}+\varepsilon, \varepsilon_{3}-\varepsilon_{4}, \varepsilon_{4}-\varepsilon_{5}, \varepsilon_{5}-\varepsilon_{6}, \varepsilon_{1}+\varepsilon_{2}+\varepsilon_{6}+\varepsilon-$ система простых корней). Имеет место случай (i). Таким образом,

$$
\operatorname{sep}\left(\mathrm{E}_{6}\right) \leqslant 2\left|W_{\mathrm{A}_{5}}^{\mathrm{sep}}\right|+2=242 .
$$

(7) Пусть $\Delta$ - система корней типа $\mathrm{E}_{7}$. Выберем $\alpha=\varepsilon_{1}-\varepsilon_{2}$. Тогда $\Delta_{\alpha}=\mathrm{D}_{6}\left(\varepsilon_{5}+\right.$ $\varepsilon_{6}+\varepsilon_{7}+\varepsilon_{8}, \varepsilon_{3}-\varepsilon_{4}, \varepsilon_{4}-\varepsilon_{5}, \varepsilon_{5}-\varepsilon_{6}, \varepsilon_{6}-\varepsilon_{7}, \varepsilon_{1}+\varepsilon_{2}+\varepsilon_{7}+\varepsilon_{8}-$ система простых корней). Имеет место случай (i). Таким образом,

$$
\operatorname{sep}\left(\mathrm{E}_{7}\right) \leqslant 2\left|W_{\mathrm{D}_{6}}^{\mathrm{sep}}\right|+2=2^{9} \cdot 3^{2}+2=4610 .
$$

(8) Пусть $\Delta$ - система корней типа $\mathrm{E}_{8}$. Выберем $\alpha=\varepsilon_{1}-\varepsilon_{2}$. Тогда $\Delta_{\alpha}=\mathrm{E}_{7}\left(\varepsilon_{6}+\varepsilon_{7}+\varepsilon_{8}\right.$, $\varepsilon_{3}-\varepsilon_{4}, \varepsilon_{4}-\varepsilon_{5}, \varepsilon_{5}-\varepsilon_{6}, \varepsilon_{6}-\varepsilon_{7}, \varepsilon_{7}-\varepsilon_{8}, \varepsilon_{1}+\varepsilon_{2}+\varepsilon_{8}-$ система простых корней). Имеет место случай (i). Таким образом,

$$
\operatorname{sep}\left(\mathrm{E}_{8}\right) \leqslant 2\left|W_{\mathrm{E}_{7}}^{\text {sep }}\right|+2=9222 .
$$


(9) Пусть $K$ - группа отражений $H_{3}$ (здесь и далее используются обозначения и терминология [3]). Выберем в качестве корня $\alpha$ старший корень. Тогда $W_{\alpha}=W_{\mathrm{A}_{2}}$ и, тем самым,

$$
\left|W_{H_{3}}^{\mathrm{sep}}\right| \leqslant 2\left|W_{A_{2}}^{\mathrm{sep}}\right|+2=14 .
$$

(10) Пусть $K$ - группа отражений $H_{4}$. В качестве корня $\alpha$ выбирается старший корень. Тогда $W_{\alpha}=H_{3}$,

$$
\left|W_{H_{4}}^{\mathrm{sep}}\right| \leqslant 2\left|W_{H_{3}}^{\mathrm{sep}}\right|+2=30 .
$$

ЗАмечАние 8. Рассмотрим старший корень $\alpha_{0}$ и расширенную диаграмму Дынкина. Диаграммы, соответствующие системам $\Delta_{\alpha}$, получаются следующим образом: необходимо выкинуть из расширенной диаграммы $\alpha_{0}$ и все корни, к нему примыкающие. Докажем это. Заметим, что $\Delta_{\alpha_{0}}^{+} \subset \Delta^{+}$. Также те простые корни, которые не были выкинуты из диаграммы, остаются неразложимыми и, тем самым, будут простыми в $\Delta_{\alpha_{0}}$ (назовем их набор $\left.\Pi_{\alpha_{0}}\right)$. Докажем, что $\Pi_{\alpha_{0}}-$ система простых корней в $\Delta_{\alpha}$. Пусть есть некоторый положительный разложимый корень $\beta=\sum_{\alpha \in \Pi} c_{\alpha} \alpha \in \Delta$, где $c_{i} \geqslant 0$, который является простым в $\Delta_{\alpha}$. Тогда

$$
\left\langle\beta, \alpha_{0}\right\rangle=\sum_{\alpha \in \Pi} c_{\alpha}\left\langle\alpha, \alpha_{0}\right\rangle=0 .
$$

Но так как $\alpha_{0}$ - старший корень, то он лежит в камере Вейля и, значит, $\left\langle\alpha, \alpha_{0}\right\rangle \geqslant 0$. Из предыдущего равенства мы получаем, что $c_{\alpha} \neq 0$ выполнено, только если $\left\langle\alpha, \alpha_{0}\right\rangle=0$, ибо иначе для всех корней $\alpha$, входящих в разложение $\beta$, выполнено $\alpha \in \Pi_{\alpha_{0}}$, что противоречит неразложимости корня $\beta$.

Авторы благодарят В.Л. Попова за постановку задачи и внимание к работе и Э.Б. Винберга за обсуждение ряда вопросов.

\section{СПИСОК ЦИТИРОВАННОЙ ЛИТЕРАТУРЫ}

[1] V.L. Popov, Tensor product decompositions and open orbits in multiple flag varieties, arXiv: math.RT/0609003. [2] Э. Б. Винберг, А.Л. Онищик, Семинар по группам Ли и алгебраическим группам, УРСС, Москва, 1995. [3] J. Humphreys, Reflection Groups and Coxeter Groups, Cambridge Studies in Advanced Mathematics, 29, Cambridge Univ. Press, Cambridge, 1990.

\section{В. Жгун}

Поступило

Московский государственный

05.12 .2006

университет им. М. В. Ломоносова

E-mail: zhgoon@mail.ru

\section{Д. Миронов}

Математический институт им. В. А. Стеклова РАН

E-mail: mironovd@mccme.ru 